\title{
Influence of interface on the formation process of polymer coatings on metal
}

\author{
O. G. Maksimova*, A. V. Maksimov and A. I. Moiseeva \\ Cherepovets State University, Vologda Region \\ Cherepovets, 162600 Russian Federation, Russia \\ *og62@mail.ru
}

\author{
Received 25 November 2015; Revised 4 January 2016; Accepted 21 January 2016; Published 23 March 2016
}

\begin{abstract}
The purpose of this work is in development of the model that allows to investigate the conformations of macromolecules near the interface "dielectric-metal" depending on the conditions of formation of the polymer coating. In the modified model of "sticky tape", one part of macromolecule is anchored to the metal surface while the other can be elongated due to effective mean (molecular) field of dipolar type formed by free ends of other chains. The dynamic Monte-Carlo method for Langmuir's model is used for calculation of adhesion force taking into account the interaction energy of monomers with the metal surface. It is shown that conformation of polymer chain is defined by temperature conditions of its formation. The obtained results are confirmed by the data of production tests on polymer coatings in JSC "Severstal".

Keywords: Polymer coatings; order parameter; Monte-Carlo method; Langmuir's model; mean field; adhesion energy.
\end{abstract}

\section{Introduction}

Study of the physico-chemical properties of surface polymer structures (e.g., films, layers and membranes) is not only of theoretical but also practical significance due to their unique characteristic importance for many applications. The phenomenon of the adhesion of polymer chains on surfaces plays an important role in such technological processes, e.g., a formation of protective coatings for sheet steel products for the worldwide construction and car industry. ${ }^{1}$ Other applications with tremendous importance are the reinforcement of rubber networks by addition of carbon black filler particles. The adhesion of polymers is widely used for stabilization of flocculating agents for colloidal dispersions, ${ }^{2}$ biocompatibility, ${ }^{3,4}$ chromatographic separation, ${ }^{5}$ etc. The adsorption of polymer molecules on cell membranes and organelles, soils and colloidal aggregates are some examples in biological systems.

Recently, many excellent experimental techniques were developed to study the polymer adsorption. The relative experimental apparatus such as atomic force microscopy (AFM) and scanning electronic microscope ${ }^{6}$ have been used widely to study the adsorption phenomenon. This apparatus allows to determine the structure of polymer layers near the solid surface and develop model conceptions for description of the conformations of polymer chains adsorbed on the surface.

Solving the problem of macromolecule adsorption creates a foundation for the theory of polymer adhesion at interfaces. Numerous experimental and theoretical studies have been devoted to the adsorption phenomenon of polymer chains at solid surfaces. ${ }^{7-18}$ Many scientists have used various parameters such as the surface coverage, layer thickness, average bound fraction of adsorbed polymer segments and polymer volume fraction profile normal to the interface. Control of such macroscopic characteristics of the polymer covering as thickness, density and strength during its formation is also an actual problem by the production of polymer coatings of metals. ${ }^{1}$ On one hand, thick films are less elastic, ${ }^{1}$ on the other hand, thin films do not serve as protective barrier from corrosion. Besides, the film thickness defines the cost of the product.

Conformation of polymer chains adsorbed on a surface is far different from that in the bulk systems. Klushin and Skvorzov ${ }^{19,20}$ have used the Gaussian model of a polymer chain in external field ("sticky tape" model) to analyze the first-order phase transitions in the adsorbed state. For classical low-molecular systems that undergo such phase transitions, describing both phases and investigating effects related to the finiteness of systems and intermolecular interactions is a very complicated task that can be accomplished only by means of numerical calculations and computer simulations. Elastic behavior of single polymer chain adsorbed on the attractive surface was first investigated using Monte-Carlo simulation method based on the bond fluctuation model. ${ }^{21}$ There are some special behaviors of the chain size and shape at the elongation beginning, especially for strong attractioninteraction. For example, mean fraction of adsorbed segments decreases abruptly in the region of small elongation ratio and then decreases slowly with increasing elongation ratio. ${ }^{21}$

In this work, we will study the influence of interface "dielectric-metal" and temperature conditions on the

This paper is submitted to the 2nd Russia-China Workshop on Dielectric and Ferroelectric Materials, Voronezh, Russia, Sept. 11-14, 2015.

This is an Open Access article published by World Scientific Publishing Company. It is distributed under the terms of the Creative Commons Attribution 4.0 (CC-BY) License. Further distribution of this work is permitted, provided the original work is properly cited. 
formation process of polymer coatings on metal, on conformations of the chains, the process of polymerization and adhesion. It is necessary to consider the behavior of macromolecules on metal surface as a complex task, which is solved by a number of controlling parameters.

This paper is organized as follows. In Sec. 2, we develop the modified "sticky tape" model with intermolecular interactions in the mean-field approach and analyze solutions of self-consistent equation for the order parameter - the mean elongation degree of chains. In Sec. 3, we determinate parameters of this model: values of adhesion energy, the mean field parameter and the equilibrium length of the polymer chains. The value of each of the parameters is defined not only by chemical structure of the polymer but also by thermal regime of coating formation. In Sec. 4, we focus on main results, simulation results and comparison with production test data.

\section{Model}

The structure of the contact layer between polymer and steel sheet consists of the ordered fibers, perpendicular to the steel sheet surface (Fig. 1(a)). Therefore, the model of "sticky tape" may be used for research of physico-chemical properties of such polymer coatings. In the standard "sticky tape" model, ${ }^{19,20}$ flexible polymer chain is in solution near the adsorbing surface while the other one is in external field (Fig. 1(b)).

In the modified "sticky tape" model, it is assumed that one part of macromolecule is anchored to the metal surface while the free chain part can be elongated due to the internal effective mean (molecular) field formed owing to intermolecular

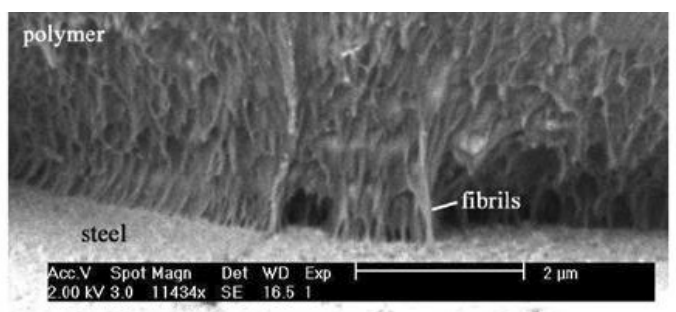

(a)

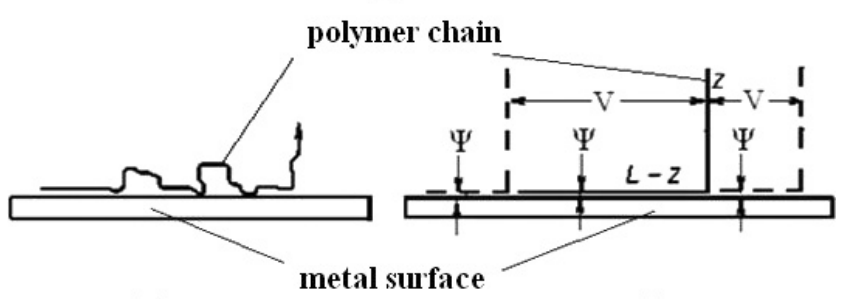

(b)

(c)

Fig. 1. (a) The image of the contact layer between the polymer coating and steel sheet, obtained by means of the scanning electronic microscope method $^{6}$; (b) The graphic representation of the usual "sticky tape" model; (c) The analytical description of the modified sticky tape model in the mean-field approach. interactions with free ends of other chains. If the length of the elongated part of the chain with the contour length $L$ is equal to $z$, its potential energy in the molecular field of dipolar type is equal to $U_{1}=-\mu W z$, where the value $\mu$ defines the order parameter equal to mean elongation degree of the polymer chain $\mu=\langle z\rangle / L$ and the parameter $V$ is the value of the meanfield interactions with other chains per a unit chain length. The other part of the chain (with length $L-z$ ) adsorbed on the surface has the energy $U_{2}=-\psi(L-z)$, where the parameter $\psi$ characterizes the adhesion energy per the length chain (Fig. 1(c)).

The self-consistency equation for the order parameter $\mu$ in the frame of the mean-field model $^{22}$ has the form:

$$
y(\mu)=\mu-1-\frac{k_{B} T}{L(\mu W-\psi) \cdot\left(\exp \left[(\mu W-\psi) \frac{L}{k_{B} T}\right]-1\right)}=0,
$$

where $T$ is the temperature of the coating. Thus, transcendental equation (1) includes all parameters of the model: $\psi$ (the value of adhesion energy), $W$ (the value of the mean-field) and $L$ (the length of the polymer chain). The values of each of the parameters will be determinate not only by chemical structure of the polymer but also by thermal regime of coating formation. These values will be calculated below in Sec. 3 .

Dependences of the function $y(\mu)$ are given in Fig. 2. It is seen that at small values of the parameter of the molecular field ( $W<W_{c}$ ) (curve 1), Eq. (1) has only one root $\mu_{11}$ not equal to zero. At sufficiently large values of molecular field $\left(W>W_{c}\right)$ (curve 3$)$, the equation has three roots $\left(\mu_{31}, \mu_{32}\right.$ and $\left.\mu_{33}\right)$. Calculations show that free energy is maximum in the point $\mu_{32}$ and minimum in points $\mu_{31}$ and $\mu_{33}$, while the deepest minimum can be observed at point $\mu_{33}$. Therefore, the root $\mu_{33}$ is the order parameter at values of the molecular field $W>W_{c}$. The value $W_{c}$ is the critical value of the meanfield parameter at which a first-order phase transition of chains from the absorbed state to elongated one exists that is confirmed by results of works. ${ }^{19-21}$

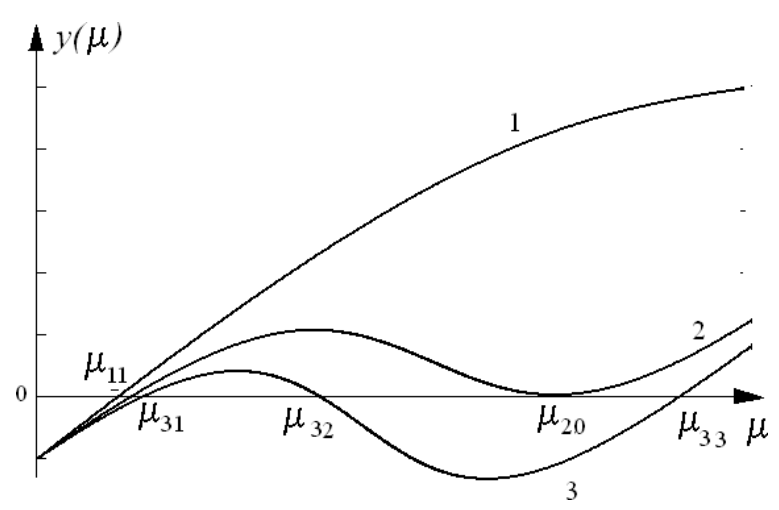

Fig. 2. The graphic solution of the self-consistency equation (1) for the mean of elongation degree of chain $\mu$ at the given value of reduced adhesion energy $\left(\psi / k_{B} T=1\right)$ and different values of the mean-field parameter: $W<W_{c}(1), W=W_{c}(2)$ and $W>W(3)$. 


\section{Determination of the Model Parameters}

The model parameters $\psi, W$ and $L$ are calculated by means of dynamic Monte-Carlo method for three modes of production tests polymer coverings which have been carried out in JSC "Severstal" (Fig. 3).

\subsection{Calculation of the parameter adhesion $\psi$}

The adhesive layer can be considered as a combination of monomers interacting with the metal sheet surface. This surface of the steel sheet may be considered as a two-dimensional rectangular lattice which consists of $N$ adsorption nodes along the $X$-axis and of $M$ nodes along the axis $Y$. Thus, the placement of the node in the lattice is defined by two numbers $i$ and $j$, and its employment is characterized by number $n_{i, j}$ which is equal to zero if the adsorption node is still free, otherwise this number is equal to the unit. The interaction energy between the different lattice nodes in the modified Langmuir's adsorption model is determined by the formula,

$$
E=-\sum_{i, j=1}^{N, M} K n_{i, j} n_{i+1, j}-\sum_{i, j=1}^{N, M} K n_{i, j} n_{i, j+1},
$$

where $K$ is the interaction constant. In Eq. (2), interactions between the nearest nodes are alone taken into account.

The relative degree of adhesion is defined as the ratio of number of adsorbed monomers to total number of lattice nodes, i.e.,

$$
\theta=(1 / N M) \sum_{i, j=1}^{N, M} n_{i, j} .
$$

We assume that the adhesion parameter $\psi$ can be presented in the form:

$$
\psi=\psi_{0} \theta,
$$

where $\psi_{0}$ is mean energy per a adsorbed monomer.

The degree of adhesion of monomers to metal is calculated by means of dynamic Monte-Carlo method on the modified Langmuir's adsorption model. ${ }^{7,23}$

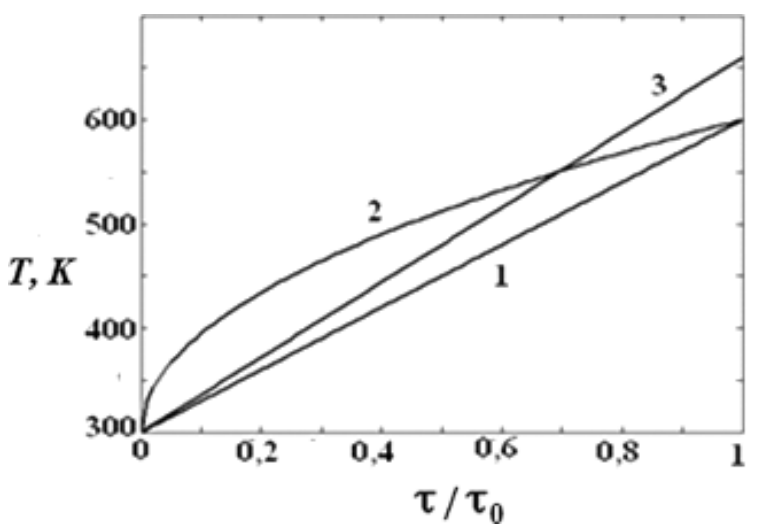

Fig. 3. The reduced time dependences of temperature regimes of heating rates of the metal sheet surface.

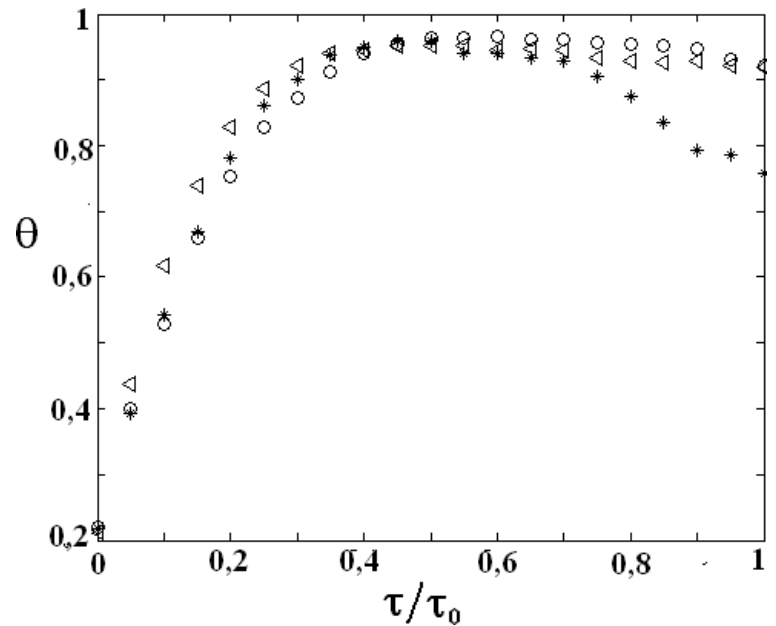

Fig. 4. The reduced time dependences of the adhesion degree of monomers to the steel surface for regimes of heating rates of the metal sheet surface indicated in Fig. 3: o(1), $\Delta_{0}(2), *(3)$ correspondingly.

At initial time $(t=0)$, it is accepted that the value $n_{i, j}=0$ for all the nodes of the lattice, that means there is no adsorption. The energy of each monomer is a random number defined by the Maxwell's distribution at the given temperature. The monomer can reach the surface of metal (that corresponds to the bottom of the potential well with the depth $E_{c}$ ), overcoming some energy barrier $E_{b}{ }^{22,24}$ Then, the electron exchange between the monomers and the metal surface is possible. It leads to rather strong chemical bonds between them, i.e. to chemical adsorption. The sum of energies $E_{c}+E_{b}$ determines the energy required for the transition of monomer from the adsorbed state (on the metal surface) into the free one.

The dependences of the adhesion coefficient at different heating rates during the surface formation calculated for modified Langmuir's model are shown in Fig. 4. By simulation, it is accepted that the maximal reduced time of coating drying $\tau / \tau_{0}=1$. It is seen that very high heating speed of metal sheet (regime 3 ) results in largest decrease of degree of adhesion of monomers.

\subsection{Calculation of the mean field parameter $W$}

By drawing of monomer paint at the metal sheet, this paint contains solvent which evaporates during the drying process. At the decrease of the solvent, the values of interchain interaction parameter $W$ increases inversely proportional to the square of the volume. ${ }^{25}$ Just as the metal sheet with the crude coating thickness $h_{1}$ moves inside the drying stove, the coating temperature changes, and its thickness decreases with the speed $\Omega$, up to the dry layer thickness $h_{2}$. The speed of evaporation of the solvent can be defined from the LambertKnudsen's equation:

$$
\Omega=k P_{0} \sqrt{\frac{2 \pi R T}{M}} \exp \left(\frac{M \lambda}{R}\left(\frac{1}{T_{0}}-\frac{1}{T}\right)\right),
$$


where $k$ is the coefficient of proportionality, $P_{0}$ is the pressure of saturated vapor of the metal sheet surface at the temperature $T_{0}=300 \mathrm{~K}, R$ is gas constant, $T$ is the temperature of the metal sheet surface, $M$ is the molar mass of the monomer, $\lambda$ is the specific heat of evaporation.

The evaporation of the solvent is followed by the decrease of the material volume: $\frac{d V}{d \tau}=\Omega$. By integrating this equation from the value $h_{1}$ to the value $h_{2}$, we obtain the dependence of the constant $W$ on the temperature $T$.

\subsection{Calculation of the equilibrium polymer chain length $L$}

Originally, by the formation of polymer coating, the rate of the chain length growth $\left(W_{1}\right)$ considerably exceeds the speed of its break $\left(W_{2}\right)$. By the increase of the quantity of monomers which enter the reaction of polymerization, the break speed increases and the chain growth stops: $\frac{d L}{d \tau}=W_{1}-W_{2}$. Therefore, in this work, it is supposed that the process of growth of the chain length is occurring until the time when the growth rate and the break one coincide, i.e., $W_{1}=W_{2}$. The rate of the chain length growth is calculated by the formula $W_{1}=A \exp \left(-\frac{E_{a}}{R T}\right)$, where $E_{a}$ is the energy of activation, $A$ is the coefficient of proportionality, the speed of break $W_{2}$ is proportional to the number of monomers which entered the reaction of polymerization, calculated by means of the classical equation of Arrhenius.

\section{Discussion of Simulation Results and Comparison with Production Tests Data}

In this work, we study the mean-field modified "sticky tape" model in which, as in the usual model, we can obtain a strict analytical description of both phases by the condition that all parameters of the model can be calculated by means of dynamic Monte-Carlo method. The developed model allows to investigate the conformations of the polymer chain near the metal surface.

The results of the simulation show that the order parameter in the polymer coating, formed by macromolecules nearly the metal surface, significantly depends on temperature conditions of coating formation. So, the thickness of the coating is affected not only by the maximum temperature of heating (the peak temperature of metal) but also by the speed of temperature change at the initial stage of coating formation. To compare these results with the experiment, it is accepted that the polymer chain elongation degree is proportional to the thickness of the obtained polymer film. Calculations show that the regime 2 (Fig. 3) gives advantage in thickness of the polymer coating about $6 \%$, in comparison with uniform heating (regime 1). Simultaneously, the uniform heating up to higher temperature (regime 3 ) gives the increase in the coating thickness only by $2 \%$. These results are confirmed for quality by the experimental data obtained from production tests for the coatings from polyester enamel implemented at JSC "Severstal" in the drying stove by means of the "coil coating" technology. The error in the comparison of simulation data and production tests is $12 \%$.

\section{Acknowledgments}

The study results are obtained at the performance of State Task No. 2014/267 from 31.01.2014, No. 1715.

\section{References}

${ }^{1}$ M. A. Benyakovskii and V. A. Maslennikov, Avtomobil'naya steel' $i$ tonkii list (Car's Steel and Thin Sheet), (Izd. Dom "Cherepovets" Cherepovets, 2007).

${ }^{2}$ D. Nanper, Polymer Stabilization of Colloidal Dispersions Academic (London, 1983).

${ }^{3}$ J. D. Andrade, Surface and Interfacial Aspects of Biomedical Polymers (Plenum, New York, 1985).

${ }^{4}$ W. W. Yau, J. J. Kirkland and D. Bly, Modern Size Exclusion Chromatography (Wiley, New York, 1979).

${ }^{5} \mathrm{~S}$. Wu, Polymer Interfaces and Adhesion (Dekker, New York, 1982).

${ }^{6}$ M. J. Van den Bosch, Interfacial delamination in polymer coated metal sheet, a numerical-experimental study. Eindhoven. Eindhoven University of Technology, University Press Facilities; 2007.

${ }^{7}$ I. Langmuir, Vapor presseres, evaporation condensation and adsorption, J. Am. Chem. Soc. 54(7), 2798 (1932).

${ }^{8}$ M. C. M. van der Sanden, H. E. H. Meijer and P. J. Lemstra, Deformation and toughness of polymeric systems: 1 . The concept of a critical thickness, Polymer, 34, 2148 (1993).

${ }^{9}$ M. Cohen-Stuart, T. Cosgrove and B. Vincent, Experimental aspects of polymer adsorption at solid/solution interfaces, $A d v$. Colloid Interface Sci. 24, 143 (1986).

${ }^{10}$ G. F. Belder, G. T. Brinke and G. Hadziioannou, Influence of anchor block size on the thickness of adsorbed block copolymer layers, Langmuir. 13, 4102 (1997).

${ }^{11} \mathrm{M}$. Xie and F. D. Blum, Adsorption and dynamics of poly(styreneb-2-vinylpyridine) on silica and alumina in toluene, Langmuir. 12, 5669 (1996).

${ }^{12}$ R. Zajac and A. Chakrabarti, Statics and dynamics of homopolymer adsorption and desorption: A Monte Carlo study, J. Chem. Phys. 104, 2418 (1996).

${ }^{13}$ T. Haliloglu and W. L. Mattice, Conformational transitions of endadsorbed triblock copolymers in a nonselective solvent, Macromol. Theory Simul. 6, 667 (1997).

${ }^{14}$ T. Haliloglu, D. C. Stevenson and W. L. Mattice, Monte Carlo simulation of the adsorption from a nonselective solvent of symmetric triblock copolymers with sticky end blocks, J. Chem. Phys. 106, 3365 (1997).

${ }^{15}$ P. Y. Lai, Statics and dynamics of adsorbed polymer chains: A Monte Carlo simulation, J. Chem. Phys. 103, 5742 (1995).

${ }^{16}$ E. A. Zheligovskaya, P. G. Khalatur and A. R. Khokhlov, Polymer chain binding with a flat adsorbent in the case of selective adsorption of segments: Monte Carlo simulation, J. Chem. Phys. 106, 8598 (1997).

${ }^{17}$ R. Zajac and A. Chakrabarti, Kinetics and thermodynamics of end-functionalized polymer adsorption and desorption processes, Phys. Rev. E 49, 3069 (1994).

${ }^{18}$ Y. Rouault, From comb polymers to polysoaps: A Monte Carlo attempt, Macromol. Theory Simul. 7, 359 (1998). 
${ }^{19}$ L. I. Klushin, A. M. Skvorzov and A. A. Gorbunov, Adsorption of a macromolecule in an external field: An exactly solvable model with bicritical behavior, Phys. Rev. E 56, 1511 (1997).

${ }^{20}$ L. I. Klushin, A. M. Skvortsov and F. A. M. Leemarkers, Exactly solvable model with stable and metastable states for a polymer chain near an adsorbing surface, Phys. Rev. E 66, 036114 (2002).

${ }^{21}$ J. Chen, L. Zhang and J. Cheng, Elastic behavior of adsorbed polymer chains, J. Chem. Phys. 121, 11481 (2004).

${ }^{22}$ O. G. Maksimova, O. S. Baruzdina and A. V. Maksimov, Simulation of electrical properties of polymer coatings on metal surface, Ferroelectrics, 476(1), 12 (2015).
${ }^{23}$ O. G. Maksimova and A. V. Maksimov, Theoretical investigation of adhesion properties of polymer coatings of metals, Macromol. Symp. 346, 59 (2014).

${ }^{24} \mathrm{M}$. Prutton, Introduction to Surface Physics (Clarendon Press, Oxford; 1994).

${ }^{25}$ S. Chandrasekhar, Liquid Crystals (Cambridge University. Press, Cambridge, 1977). 